TITLE:

\title{
A Toy-Model Study of the Grazing Collisions in the Kinetic Theory
}

$\operatorname{AUTHOR}(\mathrm{S})$ :

Takata, Shigeru

CITATION:

Takata, Shigeru. A Toy-Model Study of the Grazing Collisions in the Kinetic Theory. Journal of Statistical Physics 2015, 160(3): 770-792

ISSUE DATE:

2015-08

URL:

http://hdl.handle.net/2433/261187

RIGHT:

This is a post-peer-review, pre-copyedit version of an article published in 'Journal of Statistical Physics'. The final authenticated version is available online at: https://doi.org/10.1007/s10955-015-1259-0.; この論文は出版社版でありま せん。引用の際には出版社版をご確認ご利用ください。; This is not the published version. Please cite only the published version. 
Journal of Statistical Physics manuscript No.

(will be inserted by the editor)

\title{
A toy-model study of the grazing collisions in the kinetic theory
}

Shigeru Takata

Version: August 29, 2014

\begin{abstract}
As a toy model study for the grazing collision effect in the Boltzmann equation, a spatially homogeneous problem for the two-dimensional Lorentz gas from the initial data with a jump discontinuity is investigated. The collision kernel of our model has the same angular singularity as the Boltzmann collision kernel. Thanks to the simplicity of the model, the jump propagation and its regularization are clearly seen, depending on whether the collision cross-section is finite or infinite. Three types of behavior are observed: a jump propagation, a jump propagation accompanied with a divergent derivative, and no jump propagation. For the model with a critical parameter, the switching from the second type to the last is observed.
\end{abstract}

Keywords Lorentz gas, grazing collisions, kinetic theory, Boltzmann equation

PACS $51.10 .+\mathrm{y}, 47.45 .-\mathrm{n}$

Mathematics Subject Classification (2010) 82B40, 76P05, 82C40

\section{Introduction}

In the present paper, as a toy model for the grazing collision effect in the Boltzmann equation, we shall consider the Lorentz gas, especially its two-dimensional version, and study a spatially homogeneous problem from the initial data with a jump discontinuity. The potential model that we employ is simple but mimics the important feature of infinite-range potentials, as well as finite-range potentials, for the Boltzmann equation.

The collision integral of the Boltzmann equation is composed of the so-called gain and loss terms. Usually, the collision integral is considered for molecular models with a finite-range intermolecular potential, and the gain and the loss term are derived separately. The hard-sphere model is a typical example. For such models, since the potential range is finite, so is the collision cross-section; the mean-free-path or the collision frequency is defined without ambiguity.

Department of Aeronautics and Astronautics \& Advanced Research Institute of Fluid Science and Engineering, Kyoto University, Kyoto-daigaku-Katsura, Nishikyo-ku, Kyoto 6158540, Japan

E-mail: takata.shigeru.4a@kyoto-u.ac.jp 
The extension to the infinite-range-potential model is done by first restricting that potential in a finite range (or assuming the impact parameter to be finite), obtaining the gain and the loss term separately, and then letting the range to be infinite $[1,2]$. After the last limiting process, however, the gain and the loss term neither remain finite; only the collision integral as a whole remains finite. The divergence of individual terms is due to the naive counting of the collision events: even the particle pairs with an extremely large distance of closest approach that result in an extremely small momentum exchange are counted to collide. Such collisions with (possibly infinitesimally) small momentum exchange are referred to as the grazing collisions.

Because of the grazing collisions, the literal mean-free-path or the collision frequency diverges, like the gain and the loss term, for infinite-range potentials. However, one can introduce its effective value, thanks to the small momentum exchange in each grazing collision. For instance, the momentum-transfer cross-section, the viscosity cross-section, etc. are found in the literature [3] for determining the effective meanfree-path. These cross-sections are actually helpful to remove the conceptual difficulty in defining the mean-free-path. Nevertheless, there still exists a practical inconvenience that the collision integral should be treated as a whole for infinite-range potentials.

In the meantime, as far as the proportion of the grazing collisions is small in the total momentum exchange, one may neglect them in counting the collision events. This motivates the so-called cutoff model for the original infinite-range (or non-cutoff) potential. The cutoff size is arbitrary, but there are two ways in the literature: the radial cutoff and the angular cutoff. The former cuts off the potential at a certain radial distance from the center of molecule, while the latter cuts off the deflection angle of relative molecular velocity at a certain angle. The radial cutoff is mostly used in particle simulations like the molecular dynamics (MD), while the angular cutoff is preferred in treating the Boltzmann collision integral theoretically. The advantage of the cutoff potential is that the gain and the loss term converge separately.

Intuitively, the grazing collisions would have little influence on the main physical phenomena. However, they have an impact on the propagation of singularity at the level of the velocity distribution function. For molecular models with a laterally finite collision-cross-section, a jump discontinuity is known to propagate in a gas in various situations [2], not only in the initial-value problem but also in the initial- and boundaryvalue problem [4] and in the boundary-value problem around convex body $[5,6,7]$. On the other hand, it is clarified that the grazing collision has a regularization property in the case of infinite-range potentials. For instance, Desvillettes [8] clarified for the Kac model that the jump discontinuity of the initial data disappears immediately after the initial time (see also [9]). His result seems to motivate subsequent mathematical studies, including some numerical proposals, on the Boltzmann equation for infiniterange potentials without cutoff (see, e.g., $[10,9,11,12,13]$ and the references therein).

In view of the situation in the previous paragraph, one may think that the kinetic theory provides contradicting pictures and may wonder which is correct. In our view, however, this qualitative difference should simply come from the different modeling for the same phenomenon. A sharp variation in real physics is modeled by a steep but regular function in the case of non-cutoff potentials, while it is modeled as a jump discontinuity in the case of cutoff potentials. The main purpose of the present paper is to verify our view through a space homogeneous initial-value problem of the Lorentz gas, thereby clarifying the essential feature of the grazing collision effect.

The rest of the paper is organized as follows. We first introduce our toy model, the Lorentz gas, especially its two-dimensional version, in Sec. 2. Then, we formulate the 
space homogeneous initial-value problem in Sec. 3. This is a problem of time evolution from the discontinuous initial data. Thanks to the simplicity of our model, we can construct an explicit Fourier series expression of the solution, which will be presented in Secs. 3.1 and 3.2. The results of numerical experiments are given in Sec. 4, where the propagation/regularization of jump discontinuity are discussed in various comparisons. We further discuss some details of the propagation/regularization of singularity in Sec. 5.1 and a specific feature in the regime that the grazing collision has an appreciable portion in the total momentum exchange in Sec. 5.2. We conclude with Sec. 6.

\section{A toy model}

\subsection{Lorentz gas $[14,15]$}

Consider size-less particles with a common mass and magnitude of velocity. Assume that there randomly distribute spherical scatterers of uniform size in space and that the particles collide with them elastically. There is no inter-particle collisions because particles are size-less. Then, after a proper scaling, the behavior of particles is described by the following dimensionless kinetic equation, which is the Lorentz gas model:

$$
\begin{gathered}
f_{t}+\left(\boldsymbol{\alpha} \cdot \nabla_{\boldsymbol{x}}\right) f=\int_{|\boldsymbol{\beta}|=1} b(|\boldsymbol{\alpha} \cdot \boldsymbol{\beta}|)\left\{f\left(t, \boldsymbol{x}, \boldsymbol{\alpha}_{*}\right)-f(t, \boldsymbol{x}, \boldsymbol{\alpha})\right\} d \boldsymbol{\beta}, \\
\boldsymbol{\alpha}_{*}=\boldsymbol{\alpha}-2(\boldsymbol{\beta} \cdot \boldsymbol{\alpha}) \boldsymbol{\beta}, b(a)=a .
\end{gathered}
$$

In the above equation, $f(t, \boldsymbol{x}, \boldsymbol{\alpha})$ is the dimensionless velocity distribution function (VDF), $t$ is the dimensionless time, and $\boldsymbol{x}$ is the dimensionless position vector. $\boldsymbol{\alpha}, \boldsymbol{\alpha}_{*}$, and $\boldsymbol{\beta}$ are unit vectors, among which the former two represent the dimensionless velocity of a particle. All the notations in the present paper will be dimensionless; hereafter we shall not repeat the word "dimensionless" in every occurrence of new notations. The first term of integration on the right-hand side of (2.1) is the gain term, while the second term the loss term. In the present case, the mass and energy of the particles are conserved in collisions, while their momentum is no longer conserved. Because the magnitude of particle velocity is unity, the energy conservation is reduced to the mass conservation

$$
\rho_{t}+\operatorname{div}(\rho \boldsymbol{v})=0,
$$

where

$$
\rho=\int_{|\boldsymbol{\alpha}|=1} f d \boldsymbol{\alpha}, \quad \rho \boldsymbol{v}=\int_{|\boldsymbol{\alpha}|=1} \boldsymbol{\alpha} f d \boldsymbol{\alpha} .
$$

In the above model, the collision cross-section $\nu$ is defined well and computed as

$$
\nu \equiv \int_{|\boldsymbol{\beta}|=1} b(|\boldsymbol{\alpha} \cdot \boldsymbol{\beta}|) d \boldsymbol{\beta}=\int_{0}^{2 \pi} d \varphi \int_{0}^{\pi} d \theta \sin \theta|\cos \theta|=2 \pi .
$$

\subsection{Grazing collisions and infinite-range type potential}

In (2.1), $\boldsymbol{\alpha}_{*}$ and $\boldsymbol{\alpha}$ are the post-collision and the pre-collision velocity, respectively. Thus the grazing collision is the case $|\boldsymbol{\beta} \cdot \boldsymbol{\alpha}| \sim 0$, since $2|\boldsymbol{\beta} \cdot \boldsymbol{\alpha}|$ is the magnitude of their difference. The form $b(|\boldsymbol{\alpha} \cdot \boldsymbol{\beta}|)=|\boldsymbol{\alpha} \cdot \boldsymbol{\beta}|$ in (2.1) is due to the assumption of spherical 
scatterers with finite diameter, which corresponds to the hard-sphere model for the Boltzmann equation. The positive function $B(V,|\boldsymbol{\alpha} \cdot \boldsymbol{\beta}|)$ in the Boltzmann equation, where $V \boldsymbol{\alpha}$ is the relative velocity of colliding molecules, corresponds to our $b(|\boldsymbol{\alpha} \cdot \boldsymbol{\beta}|)$ and diverges as $|\boldsymbol{\alpha} \cdot \boldsymbol{\beta}| \rightarrow 0$ for infinite-range potentials [1,2]. For instance, for the $n$ th-inverse-power potentials ( $n=5$ is the celebrated Maxwell molecule), $B$ diverges with the rate $\left|\theta-\frac{\pi}{2}\right|^{-\frac{n+1}{n-1}}$ as $\theta \rightarrow \pi / 2$, where $\theta$ is the angle between $\boldsymbol{\alpha}$ and $\boldsymbol{\beta}$.

We shall reproduce the above feature for the Boltzmann equation by a simple extension that

$$
b(|\boldsymbol{\alpha} \cdot \boldsymbol{\beta}|)=|\boldsymbol{\alpha} \cdot \boldsymbol{\beta}|^{\gamma},
$$

where $-3<\gamma \leq 1$. The collision cross-section $\nu$ then reads

$$
\nu \equiv \int_{|\boldsymbol{\beta}|=1} b(|\boldsymbol{\alpha} \cdot \boldsymbol{\beta}|) d \boldsymbol{\beta}=\int_{|\boldsymbol{\beta}|=1}|\boldsymbol{\alpha} \cdot \boldsymbol{\beta}|^{\gamma} d \boldsymbol{\beta},
$$

which remains finite only for $\gamma>-1$. When $-3<\gamma \leq-1$, although $\nu$ as well as the gain and loss terms diverge, the collision integral remains finite as a whole as far as $f$ is smooth. ${ }^{1}$ The parameter range $-3<\gamma \leq-1$ corresponds to infinite-range potentials for the Boltzmann equation. We call the model in this $(-3<\gamma \leq-1)$ and the other $(\gamma>-1)$ range the infinite-range and the finite-range type, respectively. $\gamma=-3 / 2$ corresponds to the Maxwell molecule and $\gamma=-3$ the Coulomb potential. The collision integral no longer converges for $\gamma \leq-3$.

Before going further, we remark that the collision integral can be transformed as

$$
\begin{aligned}
& \int_{|\boldsymbol{\beta}|=1}|\boldsymbol{\alpha} \cdot \boldsymbol{\beta}|^{\gamma}\left\{f\left(t, \boldsymbol{x}, \boldsymbol{\alpha}_{*}\right)-f(t, \boldsymbol{x}, \boldsymbol{\alpha})\right\} d \boldsymbol{\beta} \\
= & \int_{\left|\boldsymbol{\alpha}_{*}\right|=1}\left(\frac{1-\boldsymbol{\alpha} \cdot \boldsymbol{\alpha}_{*}}{2}\right)^{\frac{\gamma}{2}}\left\{f\left(t, \boldsymbol{x}, \boldsymbol{\alpha}_{*}\right)-f(t, \boldsymbol{x}, \boldsymbol{\alpha})\right\} d \boldsymbol{\alpha}_{*} .
\end{aligned}
$$

The derivation would be standard and is omitted here.

2.3 Two dimensional version and cutoff/non-cutoff models

We are going to consider two dimensional version of the model in Sec. 2.2, namely

$$
\begin{aligned}
& f_{t}+\left(\boldsymbol{\alpha} \cdot \nabla_{\boldsymbol{x}}\right) f=C_{\gamma}(f), \\
& \quad C_{\gamma}(f)=\int_{\left|\boldsymbol{\alpha}_{*}\right|=1}\left(\frac{1-\boldsymbol{\alpha} \cdot \boldsymbol{\alpha}_{*}}{2}\right)^{\frac{\gamma}{2}}\left\{f\left(t, \boldsymbol{x}, \boldsymbol{\alpha}_{*}\right)-f(t, \boldsymbol{x}, \boldsymbol{\alpha})\right\} d \boldsymbol{\alpha}_{*},
\end{aligned}
$$

where all the vectors are restricted in two-dimensional space and $-3<\gamma \leq 1$. The dependence of $C_{\gamma}$ on $\gamma$ does not change in quality by the reduction of dimension.

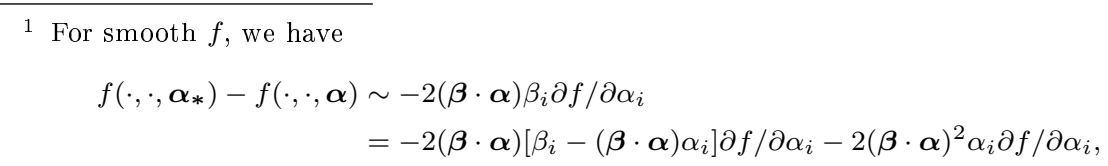

for $|\boldsymbol{\beta} \cdot \boldsymbol{\alpha}| \sim 0$ by the Taylor expansion. Since the derivatives of $f$ is evaluated at $\boldsymbol{\alpha}$, they are independent of $\boldsymbol{\beta}$. As the result, the first term of the most right-hand side does not contribute to the integration with respect to $\boldsymbol{\beta}$. The last term reduces the degree of possible singularity from $|\boldsymbol{\beta} \cdot \boldsymbol{\alpha}|^{\gamma}$ to $|\boldsymbol{\beta} \cdot \boldsymbol{\alpha}|^{\gamma+2}$, allowing the integration down to $\gamma=-3$. 
With the polar angles $\theta$ and $\theta_{*}$ such that $\boldsymbol{\alpha}=(\cos \theta, \sin \theta)$ and $\boldsymbol{\alpha}_{*}=(\cos (\theta+$ $\left.\left.\theta_{*}\right), \sin \left(\theta+\theta_{*}\right)\right),(2.2)$ is rewritten as

$$
\begin{aligned}
f_{t}+\cos \theta f_{x} & +\sin \theta f_{y}=C_{\gamma}(f), \\
C_{\gamma}(f) & =\int_{-\pi}^{\pi}\left(\frac{1-\cos \theta_{*}}{2}\right)^{\frac{\gamma}{2}}\left\{f\left(t, x, y, \theta+\theta_{*}\right)-f(t, x, y, \theta)\right\} d \theta_{*} \\
& =\int_{\theta-\pi}^{\theta+\pi}\left|\sin \frac{\phi-\theta}{2}\right|^{\gamma}\{f(t, x, y, \phi)-f(t, x, y, \theta)\} d \phi,
\end{aligned}
$$

where $f(t, x, y, \theta)$ is periodic in $\theta$ with period $2 \pi$ ( $2 \pi$-periodic, for short). This is the non-cutoff model.

In order to study the effect of grazing collisions, we need a cutoff model such that the collisions with small deflection angle $\boldsymbol{\beta} \cdot \boldsymbol{\alpha} \sim 0$ are omitted. We introduce a positive constant $\epsilon(\geq 0)$ against the angle $\theta_{*}$ between $\boldsymbol{\alpha}$ and $\boldsymbol{\alpha}_{*}$ to make the desired model by replacing $C_{\gamma}$ with $C_{\gamma, \epsilon}$ below:

$$
\begin{aligned}
C_{\gamma, \epsilon}(f) & =\left\{\int_{-\pi}^{-\epsilon}+\int_{\epsilon}^{\pi}\right\}\left(\frac{1-\cos \theta_{*}}{2}\right)^{\frac{\gamma}{2}}\left\{f\left(t, x, y, \theta+\theta_{*}\right)-f(t, x, y, \theta)\right\} d \theta_{*} \\
& =\left\{\int_{\theta-\pi}^{\theta-\epsilon}+\int_{\theta+\epsilon}^{\theta+\pi}\right\}\left|\sin \frac{\phi-\theta}{2}\right|^{\gamma}\{f(t, x, y, \phi)-f(t, x, y, \theta)\} d \phi .
\end{aligned}
$$

Once the non-zero cutoff $(\epsilon>0)$ is introduced, the collision frequency becomes finite. The gain and the loss term are allowed to be dealt with separately as $C_{\gamma, \epsilon}(f)=$ $C_{\gamma, \epsilon}^{+}(f)-\nu_{\gamma, \epsilon} f$, where $C_{\gamma, \epsilon}^{+}$and $\nu_{\gamma, \epsilon}$ are defined as

$$
\begin{aligned}
C_{\gamma, \epsilon}^{+}(f) & \equiv\left\{\int_{-\pi}^{-\epsilon}+\int_{\epsilon}^{\pi}\right\}\left(\frac{1-\cos \theta_{*}}{2}\right)^{\frac{\gamma}{2}} f\left(t, x, y, \theta+\theta_{*}\right) d \theta_{*} \\
& =\left\{\int_{\theta-\pi}^{\theta-\epsilon}+\int_{\theta+\epsilon}^{\theta+\pi}\right\}\left|\sin \frac{\phi-\theta}{2}\right|^{\gamma} f(t, x, y, \phi) d \phi, \\
\nu_{\gamma, \epsilon} & \equiv\left\{\int_{-\pi}^{-\epsilon}+\int_{\epsilon}^{\pi}\right\}\left(\frac{1-\cos \theta_{*}}{2}\right)^{\frac{\gamma}{2}} d \theta_{*} \\
& =\left\{\int_{\theta-\pi}^{\theta-\epsilon}+\int_{\theta+\epsilon}^{\theta+\pi}\right\}\left|\sin \frac{\phi-\theta}{2}\right|^{\gamma} d \phi .
\end{aligned}
$$

Obviously, $\epsilon=0$ is just a non-cutoff model, and we shall often drop 0 like $C_{\gamma}, C_{\gamma}^{+}, \nu_{\gamma}$ for simplicity when $\epsilon=0$. Note that $C_{\gamma}^{+}$and $\nu_{\gamma}$ are defined well only for $\gamma>-1$; they diverge when $\gamma \leq-1$.

\section{Initial-value problem from discontinuous data}

From now on, we focus on the space-homogeneous initial-value problem for the twodimensional Lorentz gas:

$$
\begin{gathered}
f_{t}=C(f), \quad \text { I.C. } f(0, \theta)=g(\theta), \\
C(f)= \begin{cases}C_{\gamma}(f), & \text { for non-cutoff potential, } \\
C_{\gamma, \epsilon}(f), & \text { for cutoff potential. }\end{cases}
\end{gathered}
$$


Here the initial data $g(\theta)$ is $2 \pi$-periodic in $\theta$. We are interested in the case that $g$ has a jump discontinuity at $\theta=0$. Before showing the details of analyses in Secs. 3.1 and 3.2, we present preliminary arguments that should make clear the points and ideas behind.

Let us start with the finite-range type potential: $\gamma>-1$. Since $\nu_{\gamma}$ is finite, the gain and the loss term can be treated separately. Since $C_{\gamma}^{+}$is an integral operator, the gain term $C_{\gamma}^{+}(f)$ is continuous. The time evolution of the jump discontinuity at $\theta=0$ is then described by

$$
[f]_{t}=-\nu_{\gamma}[f], \quad \text { I.C. }[f](t=0)=g\left(0_{+}\right)-g\left(0_{-}\right),
$$

where $[f] \equiv f\left(t, 0_{+}\right)-f\left(t, 0_{-}\right)$. This is easily solved to give

$$
f\left(t, 0_{+}\right)-f\left(t, 0_{-}\right)=\left[g\left(0_{+}\right)-g\left(0_{-}\right)\right] \exp \left(-\nu_{\gamma} t\right),
$$

showing that the jump discontinuity propagates in time though decays exponentially. On the other hand, for the infinite-range type potential $\gamma \leq-1$ the collision integral is not guaranteed to converge for the data with jump discontinuity. This is because the Taylor expansion in the footnote 1 is not allowed. The weak formulation, which was introduced in [8] for the non-cutoff Kac equation, serves in this case. This formulation reads in our case

$$
\begin{array}{r}
\frac{d}{d t} \int_{-\pi}^{\pi} \Omega(\theta) f(t, \theta) d \theta=-\int_{-\pi}^{\pi} b_{\gamma}^{\Omega}(\theta) f(\cdot, \theta) d \theta, \\
\text { I.C. } f(0, \theta)=g(\theta), \\
b_{\gamma}^{\Omega}(\theta) \equiv \int_{-\pi}^{\pi}[\Omega(\theta)-\Omega(\phi)]\left|\sin \frac{\theta-\phi}{2}\right|^{\gamma} d \phi,
\end{array}
$$

with $\Omega(\theta)$ being test functions. Equation (3.2) is obtained from the cutoff potential first by transforming $\int_{-\pi}^{\pi} \Omega(\theta) C_{\gamma, \epsilon}(f) d \theta$ into the form

$$
\begin{aligned}
& \int_{-\pi}^{\pi} \Omega(\theta) C_{\gamma, \epsilon}(f) d \theta=-\int_{-\pi}^{\pi} b_{\gamma, \epsilon}^{\Omega}(\theta) f(\cdot, \theta) d \theta, \\
& b_{\gamma, \epsilon}^{\Omega}(\theta)=\int_{-\pi}^{\pi} \chi_{\epsilon}(\theta-\phi) \chi_{\epsilon}(\theta-\phi-2 \pi) \chi_{\epsilon}(\theta-\phi+2 \pi)[\Omega(\theta)-\Omega(\phi)]\left|\sin \frac{\theta-\phi}{2}\right|^{\gamma} d \phi, \\
& \chi_{\epsilon}(\theta)= \begin{cases}1, & |\theta|>\epsilon, \\
0, & \text { otherwise, }\end{cases}
\end{aligned}
$$

using the periodicity of the integrand and then by taking the limit $\epsilon \rightarrow 0$. This is the process parallel to the derivation of the Boltzmann collision integral for infinite range potentials. Here the important observation is that the factor $\left|\sin \frac{\theta-\alpha}{2}\right|^{\gamma}$ is combined with the difference of the test function $[\Omega(\alpha)-\Omega(\theta)]$ in $b_{\gamma}^{\Omega}$, allowing it defined well even for $-3<\gamma \leq-1$ (remember the argument in the footnote 1 ). Thus (3.2) admits $f$ with a jump discontinuity and will be the base of the subsequent analyses.

3.1 Fourier series and Gibbs phenomenon

The weak formulation (3.2) is particularly compatible with the Fourier series. Indeed, with the Fourier coefficients $f_{n}(t)$ and $g_{n}$ defined by

$$
f_{n}(t)=\frac{1}{2 \pi} \int_{-\pi}^{\pi} f(t, \theta) e^{-i n \theta} d \theta, \quad g_{n}=\frac{1}{2 \pi} \int_{-\pi}^{\pi} g(\theta) e^{-i n \theta} d \theta
$$



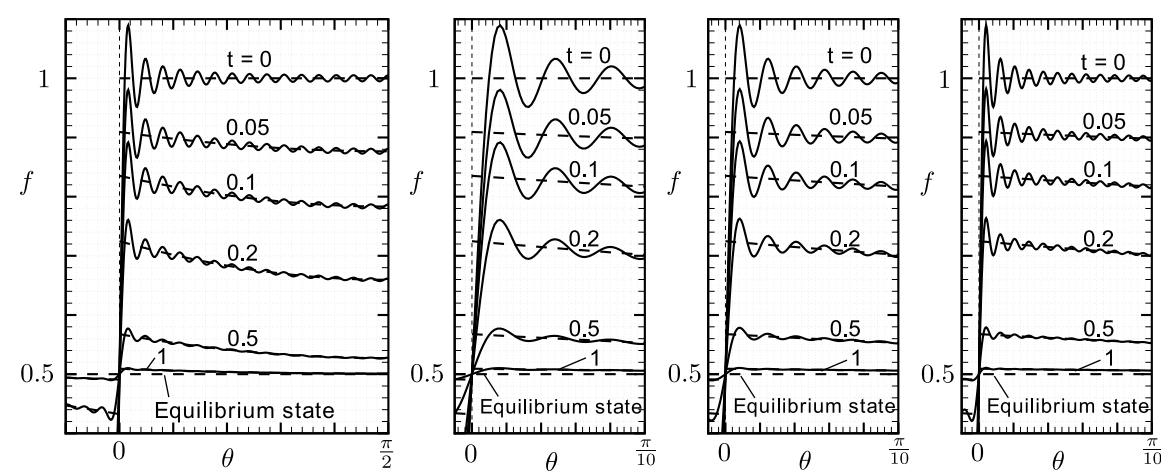

Fig. 3.1 Fourier series (3.5) and Gibbs oscillation for the initial data (3.6) for the hard-disk potential without cutoff $(\gamma=1$ and $\epsilon=0)$. Solid lines in (a) and (b) indicate (3.5) truncated at $n=61$, those in (c) indicate (3.5) truncated at $n=121$, and those in (d) indicate (3.5) truncated at $n=241$. Panels (b)-(d) show a close-up of the region $-\pi / 100<\theta<\pi / 10$. Dashed lines indicate (4.2) truncated at $m=30$. Thanks to the symmetry, only the quarter of full angle range $(0<\theta<\pi / 2)$ is shown in (a).

where $n$ is the integer, (3.2) with $\Omega=\frac{1}{2 \pi} e^{-i n \theta}$ is recast into

$$
\begin{aligned}
\frac{d f_{n}}{d t} & =-b_{\gamma}^{n} f_{n}, \quad \text { I.C. } f_{n}(0)=g_{n}, \\
b_{\gamma}^{n} & =\int_{-\pi}^{\pi}\left(1-e^{-i n(\phi-\theta)}\right)\left|\sin \frac{\theta-\phi}{2}\right|^{\gamma} d \phi=8 \int_{0}^{\pi / 2} \sin ^{2} n \psi|\sin \psi|^{\gamma} d \psi,
\end{aligned}
$$

which is easily solved to give $f_{n}=g_{n} e^{-b_{\gamma}^{n} t}$. Therefore, noting $b_{\gamma}^{n}=b_{\gamma}^{-n}(>0)$ and $b_{\gamma}^{0}=0$, the Fourier series of $f$ is given by

$$
f(t, \theta) \approx g_{0}+\sum_{n=1}^{\infty}\left(g_{n} e^{i n \theta}+g_{-n} e^{-i n \theta}\right) e^{-b_{\gamma}^{n} t} .
$$

Some remarks would be in order. The weak formulation was motivated to study the problem with discontinuous initial data. However, the Fourier series of the initial data necessarily suffers from the Gibbs oscillation when truncated, because $g$ has a jump discontinuity. The Gibbs oscillation is observed, irrespective of the value of $\gamma$, on the right-hand side of (3.5) with $t=0$. The same should occur even for $t>0$, if the jump discontinuity propagates in time as in the case of the finite-range type potential. As an illustrative example, we show in Fig. 3.1 (solid lines) the time evolution of the truncated series of (3.5) from the following initial data:

$$
g(\theta)= \begin{cases}1, & 0<\theta<\pi \\ 0, & -\pi<\theta<0\end{cases}
$$

for the hard-disk potential $(\gamma=1)$. In viewing the result, the Gibbs oscillation may be considered to work as a detector of jump discontinuity. It would prevent, however, detailed analyses at the same time. In Sec. 3.2, we present an alternative expression to avoid the drawback. 


\subsection{Improved Fourier series}

In the present subsection, we consider an alternative Fourier series expression for cutoff potentials first and then its extension to non-cutoff potentials.

The initial-value problem of our interest reads for the cutoff potential:

$$
f_{t}=C_{\gamma, \epsilon}(f)=C_{\gamma, \epsilon}^{+}(f)-\nu_{\gamma, \epsilon} f, \quad \text { I.C. } f(0, \theta)=g(\theta) \text {. }
$$

Here we assume (i) $-3<\gamma$ and $\epsilon>0$ or (ii) $\gamma>-1$, so that $C_{\gamma, \epsilon}^{+}$and $\nu_{\gamma, \epsilon}$ are both finite. It should be noted that the latter case (ii) includes the non-cutoff potentials because $C_{\gamma}^{+}$and $\nu_{\gamma}$ are finite for $\gamma>-1$.

As is preliminarily discussed in Sec. 3, the jump discontinuity of the initial data decays in time with the rate $e^{-\nu_{\gamma, \epsilon} t}$. This observation motivates us to consider the following quantity $h(t, \theta)$

$$
h(t, \theta) \equiv f(t, \theta)-g(\theta) \exp \left(-\nu_{\gamma, \epsilon} t\right),
$$

and its initial-value problem, which reads

$$
h_{t}=C_{\gamma, \epsilon}(h)+C_{\gamma, \epsilon}^{+}(g) e^{-\nu_{\gamma, \epsilon} t}, \quad \text { I.C. } h(0, \theta)=0 .
$$

Now the initial data for $h$ and $C_{\gamma, \epsilon}^{+}(g) e^{-\nu_{\gamma, \epsilon} t}$ are both continuous, $h$ is expected to be continuous for $t>0$. We consider the Fourier series of this $h$. With the Fourier coefficients $h_{n}(t)$ defined by

$$
h_{n}(t)=\frac{1}{2 \pi} \int_{-\pi}^{\pi} h(t, \theta) e^{-i n \theta} d \theta, \quad(n: \text { integer }),
$$

(3.7) is recast, after some manipulations, into

$$
\frac{d h_{n}}{d t}=-b_{\gamma, \epsilon}^{n} h_{n}+C_{\gamma, \epsilon}^{+n}(g) e^{-\nu_{\gamma, \epsilon} t}, \quad \text { I.C. } h_{n}(0)=0,
$$

where

$$
\begin{gathered}
b_{\gamma, \epsilon}^{n}=\int_{-\pi}^{\pi} \chi \epsilon(\varphi)\left|\sin \frac{\varphi}{2}\right|^{\gamma}\left(1-e^{-i n \varphi}\right) d \varphi=8 \int_{\epsilon / 2}^{\pi / 2}|\sin \psi|^{\gamma} \sin ^{2} n \psi d \psi, \\
C_{\gamma, \epsilon}^{+n}(g) \equiv \frac{1}{2 \pi} \int_{-\pi}^{\pi} C_{\gamma, \epsilon}^{+}(g) e^{-i n \theta} d \theta=\left(\nu_{\gamma, \epsilon}-b_{\gamma, \epsilon}^{n}\right) g_{n} .
\end{gathered}
$$

Equation (3.8) is easily solved to give $h_{n}(t)=g_{n}\left(e^{-b_{\gamma, \epsilon}^{n} t}-e^{-\nu_{\gamma, \epsilon} t}\right)$. Noting $b_{\gamma, \epsilon}^{n}=$ $b_{\gamma, \epsilon}^{-n}(>0)$ and $b_{\gamma, \epsilon}^{0}=0$, the Fourier series of $h$ is given by

$$
h(t, \theta) \approx g_{0}\left(1-e^{-\nu_{\gamma, \epsilon} t}\right)+\sum_{n=1}^{\infty}\left(g_{n} e^{i n \theta}+g_{-n} e^{-i n \theta}\right)\left(e^{-b_{\gamma, \epsilon}^{n} t}-e^{-\nu_{\gamma, \epsilon} t}\right) .
$$

We have thus arrived at an alternative Fourier series expression for $f$ :

$$
\begin{aligned}
f(t, \theta) \approx g(\theta) e^{-\nu_{\gamma, \epsilon} t}+g_{0}(1 & \left.-e^{-\nu_{\gamma, \epsilon} t}\right) \\
& +\sum_{n=1}^{\infty}\left(g_{n} e^{i n \theta}+g_{-n} e^{-i n \theta}\right)\left(e^{-b_{\gamma, \epsilon}^{n} t}-e^{-\nu_{\gamma, \epsilon} t}\right) .
\end{aligned}
$$




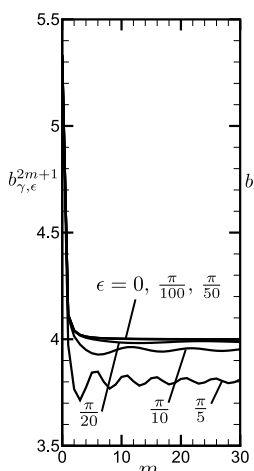

(a) $\gamma=1$

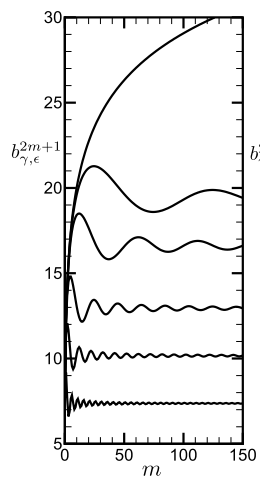

(d) $\gamma=-1$

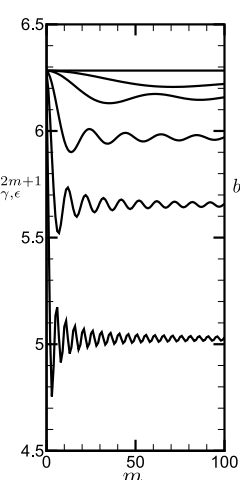

(b) $\gamma=0$

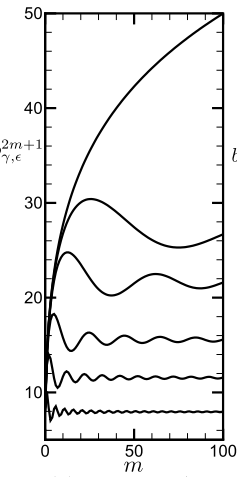

(e) $\gamma=-14 / 12$

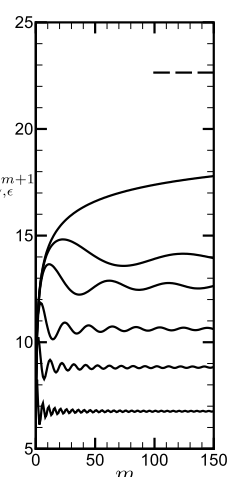

(c) $\gamma=-8 / 10$

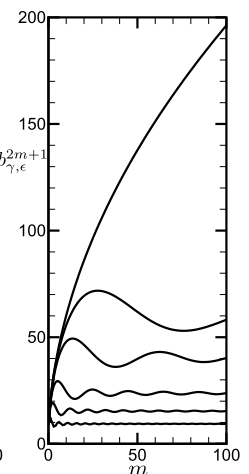

(f) $\gamma=-6 / 4$

Fig. 3.2 $b_{\gamma, \epsilon}^{2 m+1}$ for various values of $\gamma$. (a) $\gamma=1$, (b) $\gamma=0$, (c) $\gamma=-8 / 10$, (d) $\gamma=-1$, (e) $\gamma=$ $-14 / 12$, and (f) $\gamma=-6 / 4$. Solid lines indicate the results for various values of $\epsilon$ given in (a); in (b)-(f) they indicate from top to bottom the results for $\epsilon=0, \pi / 100, \pi / 50, \pi / 20, \pi / 10, \pi / 5$. Dashed line in (c) indicates the asymptote of $b_{-8 / 10}^{2 m+1}$ as $m \rightarrow \infty$. In (d)-(f), $b_{\gamma}^{2 m+1}$ tends to diverge as $m \rightarrow \infty$.

As is clear from the above form, the part $g e^{-\nu_{\gamma, \epsilon} t}$ involving the jump discontinuities is isolated in (3.10). Its Fourier series $\left[g_{0}+\sum_{n=1}^{\infty}\left(g_{n} e^{i n \theta}+g_{-n} e^{-i n \theta}\right)\right] e^{-\nu_{\gamma, \epsilon} t}$ is, instead, subtracted term by term from the Fourier series in (3.5). This term by term subtraction suppresses the possible Gibbs oscillation. Indeed, $b_{\gamma, \epsilon}^{n} \rightarrow \nu_{\gamma, \epsilon}$ as $n \rightarrow \infty,{ }^{2}$ and the factor $\left(e^{-b_{\gamma, \epsilon}^{n} t}-e^{-\nu_{\gamma, \epsilon} t}\right)$ in (3.10) improves the convergence of the series (see Fig. 3.2).

${ }^{2}$ For every fixed $\gamma$ and $\epsilon(>0)$, we have a large integer $n$ such that

$$
\begin{aligned}
\left|\nu_{\gamma, \epsilon}-b_{\gamma, \epsilon}^{n}\right|= & \left.4\left|\int_{\epsilon / 2}^{\pi / 2}\left(1-2 \sin ^{2} n \varphi\right)\right| \sin \varphi\right|^{\gamma} d \varphi|=4| \int_{\epsilon / 2}^{\pi / 2} \cos 2 n \varphi|\sin \varphi|^{\gamma} d \varphi \mid \\
= & \frac{2}{n}\left|\int_{n \epsilon}^{n \pi} \cos \phi\right| \sin \frac{\phi}{2 n}|\gamma d \phi|=\frac{2}{n}\left|\int_{n \epsilon}^{n \epsilon+2 \pi} \cos \phi\right| \sin \frac{\phi}{2 n}|\gamma d \phi| \\
& \leq \frac{2}{n} \int_{n \epsilon}^{n \epsilon+2 \pi} d \phi=\frac{4 \pi}{n} \rightarrow 0, \quad \text { as } n \rightarrow \infty
\end{aligned}
$$


Up to this point, we have assumed that (i) $-3<\gamma$ and $\epsilon>0$ or (ii) $\gamma>-1$. Beyond these parameter ranges, namely for $-3<\gamma \leq-1$ and $\epsilon=0$, we simply take the limit $\epsilon \rightarrow 0$. Then (3.10) recovers (3.5), implying that it also applies to $\epsilon=0$ beyond the assumption because (3.5) holds irrespective of whether or not $C_{\gamma}^{+n}$ and $\nu_{\gamma}$ are finite. Therefore, we adopt (3.10) for the Fourier series expression for general cases. In Fig. 3.1, (3.10) truncated at a finite number of terms is drawn with dashed lines. The Gibbs oscillation does not occur, in contrast to the truncated (3.5) (solid lines). This is the manifestation of the advantage of (3.10) over (3.5). The series convergence will be discussed in Sec. 5.1.

\section{Numerical experiments and discussions}

As already shown in Fig. 3.1, the numerical experiments are performed for the stepfunction type initial data

$$
g(\theta)= \begin{cases}1, & 0<\theta<\pi, \\ 0, & -\pi<\theta<0,\end{cases}
$$

which have jump discontinuities not only at $\theta=0$ but also at $\theta= \pm \pi$. As is easily seen from (3.10), all modes except for $n=0$ decays as $t \rightarrow \infty$, and $f$ approaches a uniform distribution, $g_{0}$ in the present case. This is no other than the equilibrium state of the Lorentz gas model with the same density as the initial data. [15]

The Fourier coefficients of the above $g$ are easily computed as

$$
g_{n}= \begin{cases}\frac{1}{2}, & n=0, \\ 0, & n: \text { (nonzero) even }, \\ \frac{1}{n \pi i}, & n: \text { odd },\end{cases}
$$

and accordingly $(3.10)$ reduces to

$$
\begin{aligned}
f(t, \theta) \approx g(\theta) e^{-\nu_{\gamma, \epsilon} t}+\frac{1}{2}\left(1-e^{-\nu_{\gamma, \epsilon} t}\right) & \\
& +\frac{2}{\pi} \sum_{m=0}^{\infty} \frac{\sin (2 m+1) \theta}{(2 m+1)}\left(e^{-b_{\gamma, \epsilon}^{2 m+1} t}-e^{-\nu_{\gamma, \epsilon} t}\right) .
\end{aligned}
$$

It is seen from the above equation that $f$ is symmetric with respect to $\theta=\pi / 2$, while $f-1 / 2$ is anti-symmetric with respect to $\theta=0$. Due to these symmetries, only the quarter part $0<\theta<\pi / 2$ of the full range of angle will be shown in the subsequent figures. Because $\nu_{\gamma, \epsilon}$ and $b_{\gamma, \epsilon}^{n}$ can be computed individually for every fixed $\gamma$ and $\epsilon$, we first perform their computations and then calculate (4.2) for various values of $\gamma$ and $\epsilon$.

4.1 Non-cutoff potential $(\epsilon=0)$

We have already seen that the jump discontinuity propagates in time in the case of the hard-disk potential $\gamma=1$ (see the dashed lines in Fig. 3.1). In Fig. 4.1, we present the results for other typical cases $\gamma=0,-8 / 10,-14 / 12$, and $-6 / 4$. In the figure, the series in (4.2) is truncated at a finite number of terms for which the series is judged to sufficiently converge. The figure clearly shows the decay of the jump at $\theta=0$ as 


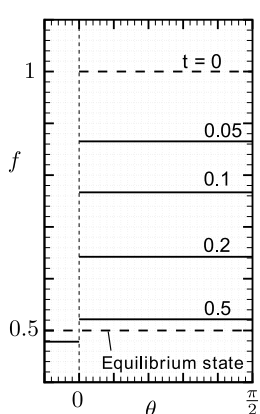

(a) $\gamma=0$

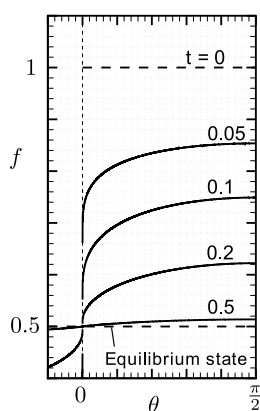

(b) $\gamma=-8 / 10$

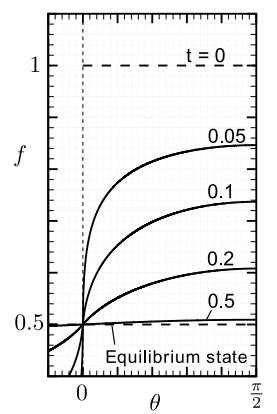

(c) $\gamma=-14 / 12$

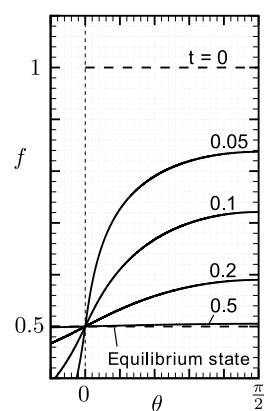

(d) $\gamma=-6 / 4$

Fig. 4.1 Fourier series (4.2) for various non-cutoff models. (a) $\gamma=0$, (b) $\gamma=-8 / 10$, (c) $\gamma=-14 / 12$, and (d) $\gamma=-6 / 4$. Series in (4.2) is truncated at $m=400$.

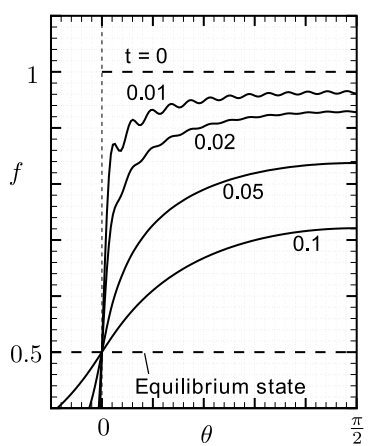

(a)

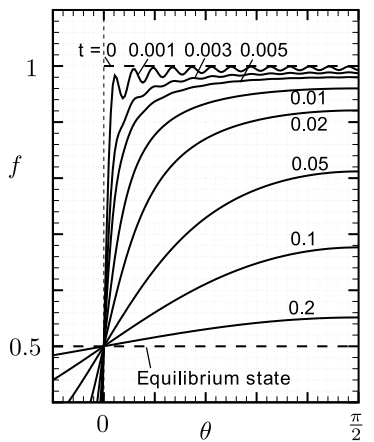

(e)

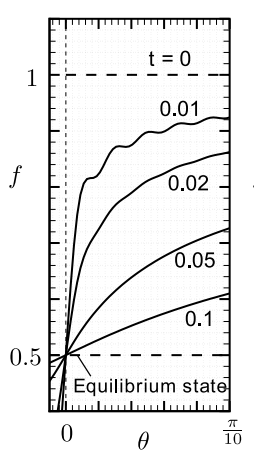

(b)

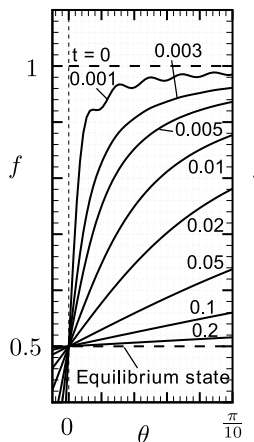

(f)

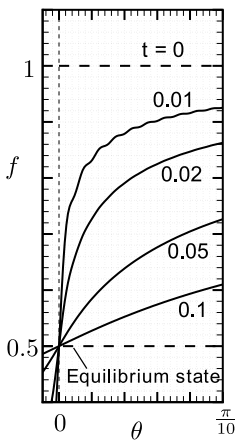

(c)

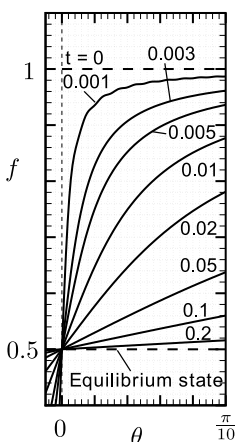

(g)

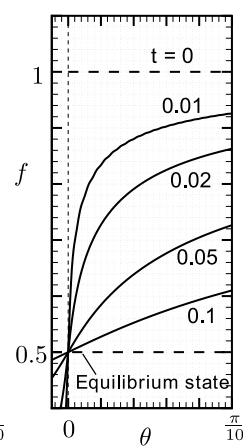

(d)

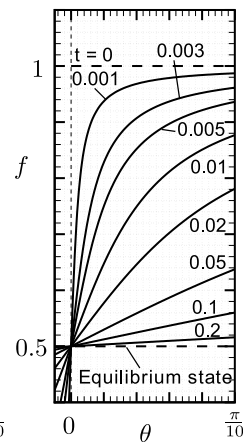

(h)

Fig. 4.2 Convergence of the series (4.2) for non-cutoff models. (a)-(d) $\gamma=-6 / 4$, (e)-(h) $\gamma=-2$. Series is truncated at $m=25$ in (a) and (e), at $m=50$ in (b) and (f), at $m=100$ in (c) and (g), and at $m=200$ in (d) and (h).

well as the overall approach of $f$ to the final equilibrium state. Note that the collision cross-section $\nu_{\gamma}$ is finite for $\gamma=0$ and $-8 / 10$, while it is infinite for $\gamma=-14 / 12$ and $-6 / 4$. For the former two, the jump discontinuity at $\theta=0$ propagates in time for $t>0$. For the latter two, it has already vanished at $t=0.05$, suggesting no propagation of discontinuity for $t>0$. 


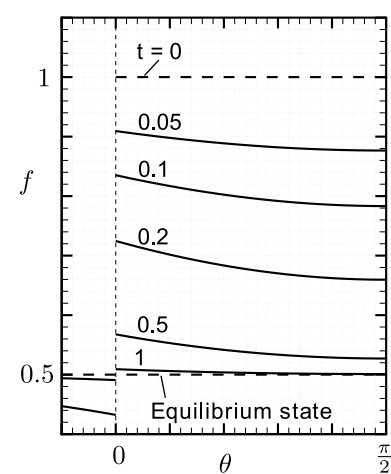

(a) $\epsilon=0$

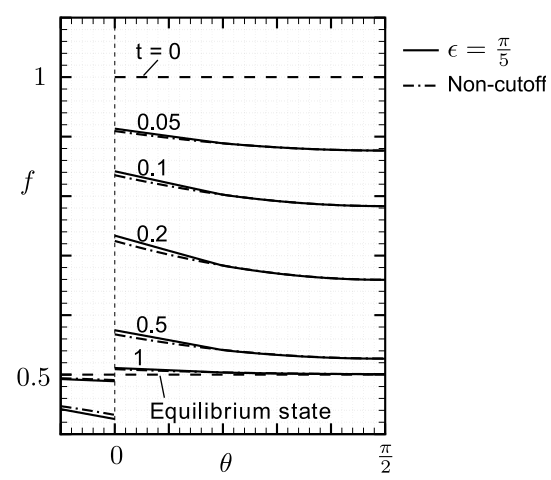

(b) $\epsilon=\pi / 5$

Fig. 4.3 Time evolution in the case of the hard-disk model $(\gamma=1)$. (a) Non-cutoff model $(\epsilon=0)$, (b) cutoff model with $\epsilon=\pi / 5$. In (b), the results for the non-cutoff model are shown by dash-dotted lines, for comparisons.

In order to make the latter clearer, we have examined the influence of the series truncation around $\theta=0$ at the earlier stage of time evolution. Figure 4.2 makes comparisons among the results for different truncations for the case with diverging $\nu_{\gamma}$ : $\gamma=-6 / 4$ and -2 . As the number of retained terms increases, the observed oscillation at every instance tends to vanish, which means no jump discontinuity at that instance. At a given instance, the series achieves better convergence for smaller $\gamma$. It should be noted, however, that in approaching the initial time $f$ becomes steeper around $\theta=0$, and accordingly more terms must be retained to achieve the practical convergence. ${ }^{3}$ Further discussions will be found in Sec. 5.1.

Going back to $\gamma=0$ and $-8 / 10$, the behavior around $\theta=0$ is quite different from each other (see Fig. 4.1), though the collision cross-section $\nu_{\gamma}$ is finite in both cases. Except for the propagation of discontinuity, $\gamma=-8 / 10$ on the whole resembles the infinite cross-section cases $\gamma=-14 / 12$ and $-6 / 4$ rather than $\gamma=0$. Such a mixed feature seems, however, reasonable in view of the smooth transition of solution via the critical value $\gamma=-1$. Further observation reveals that the solution changes steeper around $\theta=0$ for $\gamma=-8 / 10$ than for $\gamma=-14 / 12$ and $-6 / 4$. More detailed discussions will be found in Sec. 5.1.

\subsection{Cutoff effect}

We start with the simplest case $\gamma=1$, the hard-disk potential, by showing its time evolution in Fig. 4.3. Figs. 4.3(a) and (b) show the non-cutoff and the cutoff model with $\epsilon=\pi / 5$ by solid lines, respectively. In Fig. 4.3(b), the non-cutoff model is also shown by dash-dotted lines for comparisons. Here, the series in (4.2) is truncated at $m=30$ for both models. Although the setting $\epsilon=\pi / 5$ neglects collisions up to twenty percent in the deflection angle, two models agrees well; a slight difference is observed only in the range $|\theta|<\epsilon(=\pi / 5)$; the cases of $\epsilon=\pi / 10$ and $\pi / 20$ are parallel and omitted

3 In the case of $\gamma=-14 / 12$, the variation around $\theta=0$ is steeper and more terms should be retained for the practical convergence. In Fig. 4.4 that appears later, the series is truncated at $m=160,000$ (not 16,000) for $\gamma=-6 / 4$. See also the next paragraph and Sec. 5.1. 


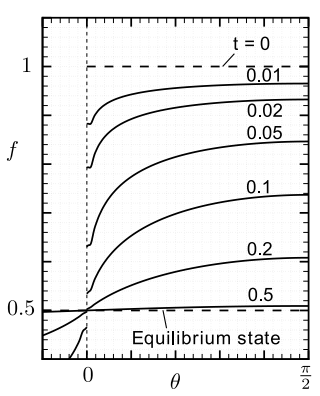

(a)

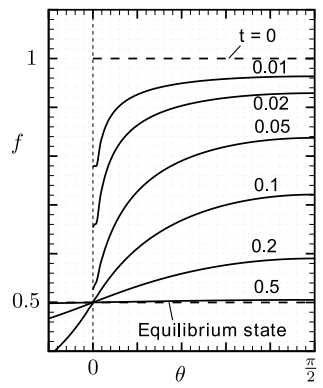

(d)

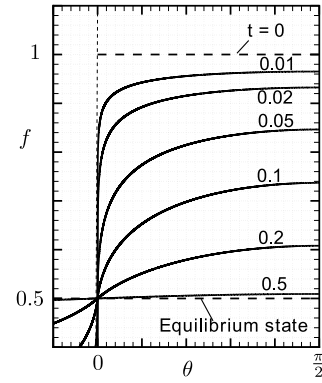

(b)

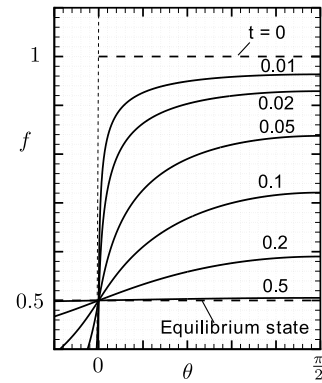

(e)

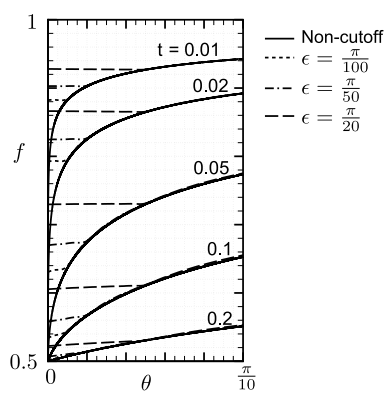

(c)

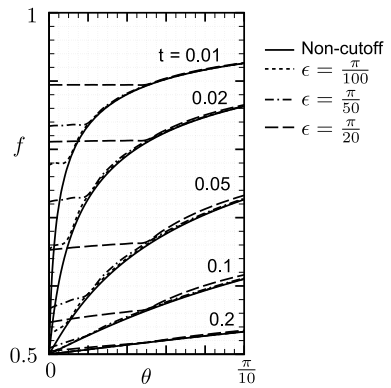

(f)

Fig. 4.4 Comparisons between the non-cutoff and the cutoff model for infinite-range type potentials: (a) $-(\mathrm{c}) \gamma=-14 / 12$ and (d) - (f) $\gamma=-6 / 4$. (a) and (d) show the results for cutoff model with $\epsilon=\pi / 100$, (b) and (e) those for non-cutoff models, and (c) and (f) a close-up of the range $0<\theta<\pi / 10$ for the non-cutoff and three cutoff $(\epsilon=\pi / 100, \pi / 50, \pi / 20)$ models. The series in (4.2) is truncated at $m=100$ for cutoff models, while it is truncated respectively at $m=160000,400$ for $\gamma=-14 / 12,-6 / 4$ for non-cutoff models.

here. The small influence of the cutoff is due to that the difference of the exponent of time decay is estimated as

$$
\begin{aligned}
\nu_{1}-\nu_{1, \epsilon} & =4 \int_{0}^{\epsilon / 2} \sin \varphi d \varphi=4\left(1-\cos \frac{\epsilon}{2}\right)=O\left(\epsilon^{2}\right), \\
b_{1}^{n}-b_{1, \epsilon}^{n} & =8 \int_{0}^{\epsilon / 2} \sin ^{2} n \varphi \sin \varphi d \varphi \\
& =2\left(2\left(1-\cos \frac{\epsilon}{2}\right)-\frac{1-\cos \frac{(2 n+1) \epsilon}{2}}{2 n+1}+\frac{1-\cos \frac{(2 n-1) \epsilon}{2}}{2 n-1}\right) \\
& =O\left(\min \left(\epsilon^{2}, \frac{\epsilon}{n}\right)\right),
\end{aligned}
$$

see Fig. 3.2(a). We have numerically confirmed that the above very limited effect applies when $\gamma>0$.

We turn to the case with the infinite collision cross-section, $\gamma=-14 / 12$ in Figs. 4.4(a)(c) and $\gamma=-6 / 4$ in Figs. 4.4(d)-(f). Figs. 4.4(a) and (d) show the results of cutoff model with $\epsilon=\pi / 100$, while Figs. 4.4(b) and (e) those of non-cutoff model. The cutoff and the non-cutoff model agree well, except that they differ considerably near $\theta=0$. To observe the difference closely, the region $0<\theta<\pi / 10$ is magnified in Figs. 4.4(c) 


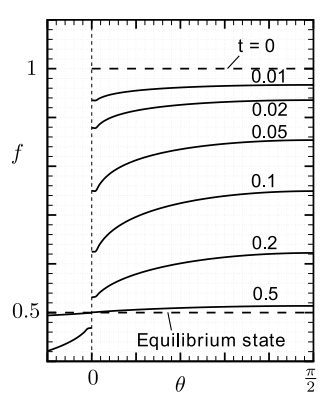

(a) $\epsilon=\pi / 100$

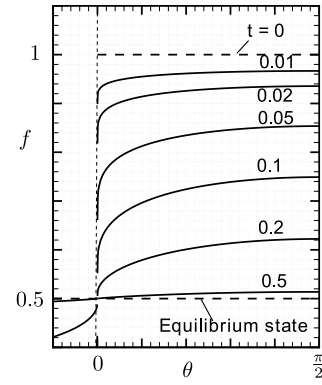

(b) $\epsilon=0$

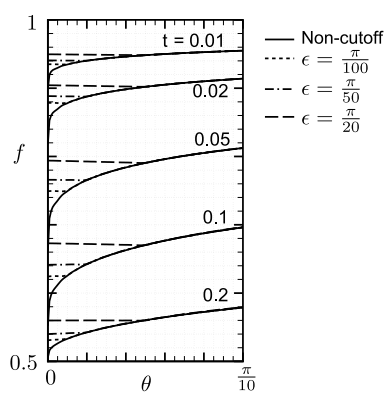

(c)

Fig. 4.5 Comparisons between the non-cutoff and the cutoff model for a finite-range type potential $(\gamma=-8 / 10)$ : (a) cutoff model with $\epsilon=\pi / 100$, (b) non-cutoff model, and (c) closeup of the range $0<\theta<\pi / 10$ for the non-cutoff and three cutoff $(\epsilon=\pi / 100, \pi / 50, \pi / 20)$ models. The series in (4.2) is truncated at $m=5000$ for non-cutoff model while at $m=100$ for cutoff models.

and (f), in which different cutoff cases $(\epsilon=\pi / 100, \pi / 50, \pi / 20)$ are compared with the non-cutoff case. The figure shows that the cutoff effect is limited inside the region $|\theta|<\epsilon$. This is the same feature as the simplest case $\gamma=1$.

It should be highly remarked that the non-cutoff model exhibits a steep variation near $\theta=0$. Indeed, in drawing Fig. 4.4(b), we retain 160, 000 (not 16,000) terms in the series of (4.2). The cutoff model effectively replaces the steep part with a jump discontinuity, allowing the series truncation at a reasonable number $m=100$ in Fig. 4.4(a).

The cutoff has a similar effect in the case of $\gamma=-8 / 10$ (Fig. 4.5). Remember that the non-cutoff model for this case has a finite collision cross-section, and accordingly the jump discontinuity at $\theta=0$ propagates with forming a steeply varying part around $\theta=0$ for $t>0$ [Figs. 4.1(b) and 4.5(b)]. The cutoff affects this part and replaces it with a jump. Again, this is well illustrated by the number of retained terms in drawing Fig. 4.5: the truncation at $m=5000$ for the non-cutoff model while that at $m=100$ for the cutoff model. ${ }^{4}$ The cutoff effect is again limited inside the region $|\theta|<\epsilon$.

To summarize, the main feature of the cutoff effect becomes clear. For $0 \leq \gamma \leq 1$ where there is no steep variation near the point of discontinuity, namely at $\theta=0$, the cutoff is rarely effective. It becomes effective when a steep variation appears around that point as in the case of $\gamma=-8 / 10,-14 / 12,-6 / 4$. The cutoff effect is essentially limited within the region $|\theta|<\epsilon$ and replaces the steep variation with a jump discontinuity. This feature, in particular for $\gamma=-14 / 12$ and $-6 / 4$, supports the viewpoint in Sec. 1 that the seemingly contradicting property - the regularization versus the singularity propagation - may be considered just as the consequence of different modeling for the same phenomenon. It should be noted, however, that we have studied here the case $\gamma>-2$ only. As mentioned in Sec. 2.2, $\gamma=-2$ is the critical case, below which the grazing collisions have an appreciable portion in the total momentum exchange. Consequently, the cutoff effect shows a different aspect for $\gamma \leq-2$, which will be discussed in Sec. 5.2.

4 As will be clear in Sec. 5.1, the truncation at $m=5000$ is not enough because $\theta$-derivative diverges. Required number of terms is larger than that for $\gamma=-14 / 12$. 


\section{Further discussions}

5.1 Series convergence for non-cutoff potentials: accompanying singularity with jump

We shall examine the convergence of the series on the right-hand side of (4.2) for the non-cutoff model, $\sum_{m=0}^{\infty} \frac{\sin (2 m+1) \theta}{2 m+1}\left(e^{-b_{\gamma}^{2 m+1} t}-e^{-\nu_{\gamma} t}\right)$, thereby clarifying the property of the steeply varying part around $\theta=0$ at which the jump discontinuity of the initial data locates (see the last two paragraphs of Sec. 4.1).

We first see the property of $b_{\gamma}^{n}$ and $\nu_{\gamma}$. In what follows, $n$ is a positive integer and $-3<\gamma \leq 1$. The integrations defining $b_{\gamma}^{n}$ and $\nu_{\gamma}$ can be carried out to yield

$$
b_{\gamma}^{n}=\kappa(\gamma)\left(1-\frac{\Gamma\left(1+\frac{\gamma}{2}\right)}{\Gamma\left(-\frac{\gamma}{2}\right)} \frac{\Gamma\left(n-\frac{\gamma}{2}\right)}{\Gamma\left(n+1+\frac{\gamma}{2}\right)}\right), \nu_{\gamma}= \begin{cases}\kappa(\gamma)(>0), & \text { for } \gamma>-1, \\ \infty, & \text { otherwise, }\end{cases}
$$

where $\Gamma$ is the gamma function and

$$
\kappa(\gamma)=2 \sqrt{\pi} \frac{\Gamma\left(\frac{1}{2}+\frac{\gamma}{2}\right)}{\Gamma\left(1+\frac{\gamma}{2}\right)} .
$$

In the above expression, $b_{-1}^{n}$ should be considered as the limiting value of $b_{\gamma}^{n}$ as $\gamma \rightarrow-1$ and is written as

$$
b_{-1}^{n}=4 \gamma_{e}+8 \ln 2+4 \Psi\left(n+\frac{1}{2}\right),
$$

where $\gamma_{e}$ is Euler's constant $(=0.5772 \ldots)$ and $\Psi$ is the digamma function $[16,17]$ (the logarithmic derivative of $\Gamma$ ). As $n \rightarrow \infty$,

$$
b_{\gamma}^{n} \sim \begin{cases}\kappa(\gamma)-2 \sqrt{\pi} \frac{\Gamma\left(\frac{1}{2}+\frac{\gamma}{2}\right)}{\Gamma\left(-\frac{\gamma}{2}\right)} n^{-\gamma-1}+O\left(n^{-\gamma-3}\right), & \text { for } \gamma \neq-1, \\ 4 \ln n+4 \gamma_{e}+8 \ln 2+O\left(n^{-2}\right), & \text { for } \gamma=-1\end{cases}
$$

Obviously from the definitions (3.4) and (2.4), $b_{\gamma}^{n} \lessgtr b_{0}^{n}(=2 \pi)=\nu_{0}$ for $\gamma \gtrless 0$.

Now we focus on the factor $\left(e^{-b_{\gamma}^{2 m+1} t}-e^{-\nu_{\gamma} t}\right) /(2 m+1)$ occurring in the series of interest. Using the above expression for $\nu_{\gamma}$ and the equality $b_{0}^{n}=\nu_{0}$, for $t>0$ and a non-negative integer $m$ we have

$$
\begin{aligned}
& \left|\frac{1}{2 m+1}\left(e^{-b_{\gamma}^{2 m+1} t}-e^{-\nu_{\gamma} t}\right)\right| \\
& = \begin{cases}\frac{e^{-b_{\gamma}^{2 m+1} t}}{2 m+1}, & \text { for }-3<\gamma \leq-1, \\
0, & \text { for } \gamma=0, \\
\frac{e^{-\kappa(\gamma) t}}{2 m+1}\left|\frac{e^{-\left(b_{\gamma}^{2 m+1}-\kappa(\gamma) t\right.}-1}{b_{\gamma}^{2 m+1}-\kappa(\gamma)}\right|\left|b_{\gamma}^{2 m+1}-\kappa(\gamma)\right|, & \text { for }-1<\gamma \leq 1 \text { and } \gamma \neq 0 .\end{cases}
\end{aligned}
$$

Here in the last equality we have also used that $b_{\gamma}^{2 m+1}-\kappa(\gamma) \neq 0$ for $0<|\gamma| \leq 1$. When $-3<\gamma<-1, b_{\gamma}^{n}>b_{0}^{n}=2 \pi>0$ and we have

$$
e^{-b_{\gamma}^{2 m+1} t}=\frac{1}{\left(b_{\gamma}^{2 m+1}\right)^{k}}\left(b_{\gamma}^{2 m+1}\right)^{k} e^{-b_{\gamma}^{2 m+1} t} \leq\left(\frac{k}{e t}\right)^{k} \frac{1}{\left(b_{\gamma}^{2 m+1}\right)^{k}},
$$

for any positive integer $k$. When $\gamma=-1$, by making use of $\Psi\left(2 m+\frac{3}{2}\right)>\ln (2 m+1)(>$ $0),{ }^{5}$ we have

$$
e^{-b_{\gamma}^{2 m+1} t}=2^{-8 t} e^{-4 \gamma_{e} t} e^{-4 t \Psi\left(2 m+\frac{3}{2}\right)}<2^{-8 t} e^{-4 \gamma_{e} t}(2 m+1)^{-4 t}
$$

5 We shall prove $\Psi\left(n+\frac{1}{2}\right)>\ln n$ for any positive integer $n$. The case $n=1$ is obvious. For $n \geq 2$, we use that 
When $-1<\gamma<0, \kappa(\gamma)-b_{\gamma}^{n}>0$ and we have

$$
\left|\frac{e^{-\left(b_{\gamma}^{2 m+1}-\kappa(\gamma)\right) t}-1}{b_{\gamma}^{2 m+1}-\kappa(\gamma)}\right|\left|b_{\gamma}^{2 m+1}-\kappa(\gamma)\right| \leq \frac{e^{c_{\gamma} t}-1}{c_{\gamma}}\left|b_{\gamma}^{2 m+1}-\kappa(\gamma)\right|,
$$

for a positive constant $c_{\gamma}$ such that

$$
c_{\gamma}= \begin{cases}2 \sqrt{\pi} \frac{\Gamma\left(\frac{1}{2}+\frac{\gamma}{2}\right)}{\Gamma\left(-\frac{\gamma}{2}\right)}, & \text { for } n \geq 2, \\ 2 \sqrt{\pi} \frac{\Gamma\left(\frac{1}{2}+\frac{\gamma}{2}\right)}{\Gamma\left(-\frac{\gamma}{2}\right)} \frac{\Gamma\left(1-\frac{\gamma}{2}\right)}{\Gamma\left(2+\frac{\gamma}{2}\right)}, & \text { for } n=1,\end{cases}
$$

by which $\kappa(\gamma)-b_{\gamma}^{n}$ is bounded from above. When $0<\gamma \leq 1, b_{\gamma}^{n}-\kappa(\gamma)>0$ and we have

$$
\left|\frac{e^{-\left(b_{\gamma}^{2 m+1}-\kappa(\gamma)\right) t}-1}{b_{\gamma}^{2 m+1}-\kappa(\gamma)}\right|\left|b_{\gamma}^{2 m+1}-\kappa(\gamma)\right| \leq\left|b_{\gamma}^{2 m+1}-\kappa(\gamma)\right| t .
$$

Thanks to (5.1),

$$
\begin{aligned}
& b_{\gamma}^{n}-\kappa(\gamma) \sim-c_{\gamma} n^{-\gamma-1}, \quad \text { for }-1<\gamma \leq 1, \gamma \neq 0, \\
& b_{\gamma}^{n} \sim-c_{\gamma} n^{-\gamma-1}, \quad \text { for }-3<\gamma<-1,
\end{aligned}
$$

for large $n$; hence, we finally have the following estimate for large $m$ :

$$
\begin{aligned}
\left|\frac{1}{2 m+1}\left(e^{-b_{\gamma}^{2 m+1} t}-e^{-\nu_{\gamma} t}\right)\right| \\
\leq \begin{cases}\left(k / e c_{\gamma}\right)^{k} t^{-k}(2 m+1)^{k(\gamma+1)-1}, & \text { for }-3<\gamma<-1, \\
2^{-8 t} e^{-4 \gamma_{e} t}(2 m+1)^{-1-4 t}, & \text { for } \gamma=-1, \\
e^{-\kappa(\gamma) t}\left(e^{c_{\gamma} t}-1\right)(2 m+1)^{-\gamma-2}, & \text { for }-1<\gamma<0, \\
0, & \text { for } \gamma=0, \\
c_{\gamma} t e^{-\kappa(\gamma) t}(2 m+1)^{-\gamma-2}, & \text { for } 0<\gamma \leq 1\end{cases}
\end{aligned}
$$

In (5.3) the definition of $c_{\gamma}$ in (5.2) has been extended to the full range $-3<\gamma \leq 1$, and $-\kappa(\gamma)+c_{\gamma}<0$ for $-1<\gamma<0$. The above estimate shows that the series $\sum_{m=0}^{\infty} \frac{\sin (2 m+1) \theta}{2 m+1}\left(e^{-b_{\gamma}^{2 m+1} t}-e^{-\nu_{\gamma} t}\right)$ converges absolutely uniformly in $\theta$ for $t>0$.

1. For any positive integer $n, \Psi\left(n+\frac{1}{2}\right)=-\gamma_{e}-2 \ln 2+2 \sum_{k=0}^{n-1} \frac{1}{2 k+1} \cdot[17,16]$

2. Since $1 / x$ is downward convex, the trapezoidal formula bounds its integral from above:

$$
\frac{1}{2}\left(1+2 \sum_{k=2}^{2 n-1} \frac{1}{k}+\frac{1}{2 n}\right) \geq \int_{1}^{2 n} \frac{d x}{x}=\ln n+\ln 2 .
$$

Then,

$$
\begin{aligned}
\Psi\left(n+\frac{1}{2}\right)-\ln n & \geq-\gamma_{e}-\ln 2+2 \sum_{k=0}^{n-1} \frac{1}{2 k+1}-\frac{1}{2}\left(1+2 \sum_{k=2}^{2 n-1} \frac{1}{k}+\frac{1}{2 n}\right) \\
& =\frac{2 n+1}{4 n(2 n-1)}-\gamma_{e}-\ln 2+\frac{1}{2}+\sum_{k=1}^{n-1} \frac{1}{2 k(2 k-1)} .
\end{aligned}
$$

Some manipulations from the first to the second line are omitted here. On the most right-hand side, the first term is positive, and the remainder is $-\gamma_{e}-\ln 2+1(>0)$ when $n=2$ and monotonically increases with $n$. The desired inequality is thus obtained. 
The term by term time-derivative of this series is also seen to converge absolutely uniformly in $\theta$ for $t>0$, and thus the right-hand side of (4.2) with $\epsilon=0$ indeed the solution $f$. Furthermore, from the decaying rate of terms in $m$, we see that $f-g e^{-\nu_{\gamma} t}$ is continuous, more precisely $C^{1}$ for $0<\gamma \leq 1, C^{\infty}$ for $\gamma=0$ and $-3<\gamma<-1$, and $C^{0}$ for $-1 \leq \gamma<0$ (see Theorem 2.6 of [18]). In particular, when $\gamma=-1$, the guaranteed regularity is improved as time goes on: for every positive integer $k, f-g e^{-\nu_{\gamma} t}$ is $C^{k}$ for $t>k / 4$.

We proceed to further studies for $-1 \leq \gamma<0$. For $-1<\gamma<0, f-g e^{-\nu_{\gamma} t}$ is guaranteed to be continuous only, because $b_{\gamma}^{n}-\nu_{\gamma} \sim n^{-\gamma-1}$ for large $n$. This slow decay of the Fourier coefficients suggests, in turn, that $f-g e^{-\nu_{\gamma} t}$ would not be differentiable at $\theta=0$ : the variation near $\theta=0$ for $\gamma=-8 / 10$ in Fig. 4.1 should be accompanied by a derivative divergence. Motivated by this observation, we introduce a $2 \pi$-periodic function for $0<\alpha<1$

$$
S(\theta ; \alpha)= \begin{cases}\theta^{\alpha}(\pi-\theta)^{\alpha}, & \text { for } 0<\theta<\pi, \\ -|\theta|^{\alpha}(\pi+\theta)^{\alpha}, & \text { for }-\pi<\theta<0,\end{cases}
$$

which is symmetric with respect to $\theta=\pi / 2$ and anti-symmetric with respect to $\theta=0$. Its Fourier series is given by

$$
\begin{aligned}
& S \approx \sum_{m=0}^{\infty} S_{2 m+1}^{\alpha} \sin (2 m+1) \theta \\
& S_{2 m+1}^{\alpha}=-\frac{2}{\pi} \int_{0}^{\pi} \theta^{\alpha}(\pi-\theta)^{\alpha} \sin (2 m+1) \theta d \theta \\
& \quad=2 \pi^{\alpha}(-1)^{m+1}(2 m+1)^{-\alpha-\frac{1}{2}} J_{\alpha+\frac{1}{2}}\left(\left(m+\frac{1}{2}\right) \pi\right) \Gamma(\alpha+1),
\end{aligned}
$$

where $J_{n}(x)$ is the Bessel function of the first kind for order $n$. For large $m, S_{2 m+1}^{\alpha}$ is estimated as

$$
\begin{aligned}
S_{2 m+1}^{\alpha} & \sim-4 \pi^{\alpha-1} \frac{\Gamma(\alpha+1) \cos \frac{\pi}{2} \alpha}{(2 m+1)^{\alpha+1}}+O\left(\frac{1}{(2 m+1)^{\alpha+2}}\right) \\
& =-4 \sqrt{\pi} \frac{\alpha}{(2 m+1)^{\alpha+1}} \frac{(2 \pi)^{\alpha-1} \Gamma\left(\frac{\alpha}{2}\right)}{\Gamma\left(\frac{1}{2}-\frac{\alpha}{2}\right)}+O\left(\frac{1}{(2 m+1)^{\alpha+2}}\right) .
\end{aligned}
$$

Here from the first to the second line we have used $\Gamma(\alpha+1)=\alpha \Gamma(\alpha)$ and $\Gamma(\alpha) \cos \frac{\pi}{2} \alpha=$ $2^{\alpha-1} \sqrt{\pi} \Gamma\left(\frac{\alpha}{2}\right) / \Gamma\left(\frac{1}{2}-\frac{\alpha}{2}\right)$, the latter of which is derived from the duplication and reflection formulas with $z=\alpha / 2$ :

$$
\Gamma(2 z)=\frac{2^{2 z}}{2 \sqrt{\pi}} \Gamma(z) \Gamma\left(z+\frac{1}{2}\right), \quad \Gamma\left(z+\frac{1}{2}\right) \Gamma\left(\frac{1}{2}-z\right)=\frac{\pi}{\cos \pi z} .
$$

The important observation is that $S_{2 m+1}^{\alpha}$ decays in $m$ with the same rate as $\left(e^{-b_{\gamma}^{2 m+1} t}-\right.$ $\left.e^{-\nu_{\gamma} t}\right) /(2 m+1)$, if $\alpha$ is chosen to be $\gamma+1$. Thus, we have

$$
\begin{gathered}
f-g e^{-\nu_{\gamma} t}-\frac{1-e^{-\nu_{\gamma} t}}{2}-\frac{2}{(2 \pi)^{\gamma+1}(\gamma+1)} S(\theta, \gamma+1) t e^{-\nu_{\gamma} t} \\
\sim e^{-\nu_{\gamma} t} \frac{2}{\pi} \sum_{m=0}^{\infty}\left(\frac{e^{\left(\nu_{\gamma}-b_{\gamma}^{2 m+1}\right) t}-1}{2 m+1}+\frac{2 \sqrt{\pi}}{(2 m+1)^{\gamma+2}} \frac{\Gamma\left(\frac{\gamma+1}{2}\right)}{\Gamma\left(-\frac{\gamma}{2}\right)} t\right. \\
\left.+O\left((2 m+1)^{-3-\gamma}\right)\right) \sin (2 m+1) \theta
\end{gathered}
$$




$$
\begin{aligned}
& \sim e^{-\nu_{\gamma} t} \frac{2}{\pi} \sum_{m=0}^{\infty}\left(\frac{\nu_{\gamma}-b_{\gamma}^{2 m+1}}{2 m+1} t+\frac{2 \sqrt{\pi}}{(2 m+1)^{\gamma+2}} \frac{\Gamma\left(\frac{\gamma+1}{2}\right)}{\Gamma\left(-\frac{\gamma}{2}\right)} t\right. \\
& \left.+O\left(\max \left((2 m+1)^{-3-\gamma},(2 m+1)^{-3-2 \gamma}\right)\right)\right) \sin (2 m+1) \theta \\
& =e^{-\nu_{\gamma} t} \sum_{m=0}^{\infty} O\left((2 m+1)^{-3-2 \gamma}\right) \sin (2 m+1) \theta,
\end{aligned}
$$

which shows that $f-g e^{-\nu_{\gamma} t}-\left(1-e^{-\nu_{\gamma} t}\right) / 2-2(2 \pi)^{-\gamma-1}(\gamma+1)^{-1} S(\theta, \gamma+1) t e^{-\nu_{\gamma} t}$ is $C^{1}$ for $-1 / 2<\gamma<0$. This estimate can be generalized to yield

$$
\begin{aligned}
f & -g e^{-\nu_{\gamma} t}-\frac{1-e^{-\nu_{\gamma} t}}{2} \\
& +\sum_{n=1}^{k-1} \frac{S(\theta, n(\gamma+1))}{(2 \pi)^{n(\gamma+1)} n !} \frac{\left[2 \sqrt{\pi} t \Gamma\left(\frac{1+\gamma}{2}\right) / \Gamma\left(-\frac{\gamma}{2}\right)\right]^{n} e^{-\nu_{\gamma} t}}{2 \sqrt{\pi} \Gamma\left(\frac{n(\gamma+1)}{2}+1\right) / \Gamma\left(\frac{1-n(\gamma+1)}{2}\right)} \\
\sim & e^{-\nu_{\gamma} t} \frac{2}{\pi} \sum_{m=0}^{\infty}\left(\sum_{n=1}^{k-1} \frac{t^{n}}{n !} \frac{\left[2 \sqrt{\pi} \Gamma\left(\frac{1+\gamma}{2}\right) / \Gamma\left(-\frac{\gamma}{2}\right)\right]^{n}}{(2 m+1)^{(\gamma+1) n+1}}\right. \\
& -\sum_{n=1}^{k-1} \frac{1}{n !} \frac{\sqrt{\pi} n(\gamma+1)}{(2 m+1)^{n(\gamma+1)+1}} \frac{\Gamma\left(\frac{n(\gamma+1)}{2}\right)}{\Gamma\left(\frac{1-n(\gamma+1)}{2}\right)} \frac{\left[2 \sqrt{\pi} t \Gamma\left(\frac{1}{2}+\frac{\gamma}{2}\right) / \Gamma\left(-\frac{\gamma}{2}\right)\right]^{n}}{2 \sqrt{\pi} \Gamma\left(\frac{n(\gamma+1)}{2}+1\right) / \Gamma\left(\frac{1-n(\gamma+1)}{2}\right)} \\
& +O\left(\max \left((2 m+1)^{-1-(\gamma+1) k},(2 m+1)^{-3-\gamma}\right)\right) \sin (2 m+1) \theta \\
= & e^{-\nu_{\gamma} t} \sum_{m=0}^{\infty} O\left(\max \left((2 m+1)^{-1-(\gamma+1) k},(2 m+1)^{-3-\gamma}\right) \sin (2 m+1) \theta,\right.
\end{aligned}
$$

which shows that for every fixed $\gamma$ the above function with $k>1 /(1+\gamma)$ is $C^{1}$ when $-1<\gamma<0$. Therefore, we conclude that, when $-1<\gamma<0$, the jump of $f$ at $\theta=0$ is accompanied by the $\theta$-derivative divergence of the same degree as the derivative of $\theta^{\gamma+1}$ (remind that $\gamma+1$ is a positive constant strictly less than 1 ).

We finally turn to the remaining case $\gamma=-1$. As already described, the estimate (5.3) suggests that the smoothness could change in time in this case. Indeed, by using $0<\Psi\left(2 m+\frac{3}{2}\right)-\ln (2 m+1) \leq(2 m+1)^{-1},{ }^{6}$ we have

$$
\left|f-\frac{1}{2}-\frac{2}{\pi} \sum_{m=0}^{\infty}\left(4 e^{\gamma_{e}}\right)^{-4 t}(2 m+1)^{-4 t-1} \sin (2 m+1) \theta\right|
$$

${ }^{6} \Psi\left(n+\frac{1}{2}\right)-\ln n>0$ is proved in footnote 5 for any positive integer $n$, where $n$ corresponds to $2 m+1$. To prove $\Psi\left(n+\frac{1}{2}\right)-\ln n \leq 1 / n$, we use the formula for $\Psi[17,16]$ that

$$
\Psi(x)=\ln x-\frac{1}{2 x}-2 \int_{0}^{\infty} \frac{t}{\left(t^{2}+x^{2}\right)\left(e^{2 \pi t}-1\right)} d t, \quad \text { for } x>0 .
$$

Then, for $F$ defined by $F(x) \equiv 1 / x-\Psi\left(x+\frac{1}{2}\right)+\ln x(x>0)$, we have

$$
\begin{aligned}
F(x) & =\frac{1}{x}+\ln x-\ln \left(x+\frac{1}{2}\right)+\frac{1}{2 x+1}+2 \int_{0}^{\infty} \frac{t}{\left(t^{2}+\left(x+\frac{1}{2}\right)^{2}\right)\left(e^{2 \pi t}-1\right)} d t \\
& \geq \frac{1}{x}+\ln x-\ln \left(x+\frac{1}{2}\right)+\frac{1}{2 x+1} \equiv G(x) \rightarrow 0 \quad \text { as } x \rightarrow \infty .
\end{aligned}
$$

By the direct calculation of $d G / d x, G(x)$ is found to be monotonically decreasing for $x>0$; thus we have $F(x) \geq G(x) \geq 0$ for $x>0$. 


$$
\begin{aligned}
& =\frac{2}{\pi}\left|\sum_{m=0}^{\infty} \frac{\sin (2 m+1) \theta}{(2 m+1)}\left(e^{-b_{-1}^{2 m+1} t}-\left(4 e^{\gamma_{e}}\right)^{-4 t}(2 m+1)^{-4 t}\right)\right| \\
& =\frac{2}{\pi}\left(4 e^{\gamma_{e}}\right)^{-4 t}\left|\sum_{m=0}^{\infty} \frac{\sin (2 m+1) \theta}{(2 m+1)^{4 t+1}}\left(e^{-b_{-1}^{2 m+1} t+\left[8 \ln 2+4 \gamma_{e}+4 \ln (2 m+1)\right] t}-1\right)\right| \\
& =\frac{2}{\pi}\left(4 e^{\gamma_{e}}\right)^{-4 t} \mid \sum_{m=0}^{\infty} \frac{\sin (2 m+1) \theta}{(2 m+1)^{4 t+1}}\left(\frac{e^{-4\left[\Psi\left(2 m+\frac{3}{2}\right)-\ln (2 m+1)\right] t}-1}{4 t\left[\Psi\left(2 m+\frac{3}{2}\right)-\ln (2 m+1)\right]}\right) \\
& \leq \frac{8}{\pi} t\left(4 e^{\gamma_{e}}\right)^{-4 t} \sum_{m=0}^{\infty} \frac{1}{(2 m+1)^{4 t+1}}\left|\Psi\left(2 m+\frac{3}{2}\right)-\ln (2 m+1)\right| \\
& \leq \frac{8}{\pi} t\left(4 e^{\gamma_{e}}\right)^{-4 t} \sum_{m=0}^{\infty} \frac{1}{(2 m+1)^{4 t+2}} .
\end{aligned}
$$

Therefore $f-\frac{1}{2}-\frac{2}{\pi} \sum_{m=0}^{\infty}\left(4 e^{\gamma_{e}}\right)^{-4 t}(2 m+1)^{-4 t-1} \sin (2 m+1) \theta$ is $C^{1}$ for $t>0$. Furthermore, because

$$
\left(4 e^{\gamma_{e}}\right)^{-4 t}(2 m+1)^{-4 t-1} \sim-\frac{\pi}{4} \frac{S_{2 m+1}^{4 t}}{\left(4 e^{\gamma_{e}} \pi\right)^{4 t} \cos (2 \pi t) \Gamma(4 t+1)},
$$

for large $m$, the $\theta$-derivative of $f$ diverges with the same degree as that of $\theta^{4 t}$ in a finite time duration $0<4 t<1$. After the critical instance $t_{\mathrm{cr}}=\frac{1}{4}, f$ is concluded to be $C^{1}$.

5.2 Momentum-transfer cross-section and cutoff for $\gamma<-2$

In Sec. 4.2, we have made comparisons between the data at a common instance. This implies that we do not pay attention to the difference of the effective collision frequency between the cutoff and the non-cutoff model.

As mentioned in Sec. 1, the effective collision frequency is well quantified by the momentum-transfer cross-section $\nu_{\gamma}^{M}$ (and $\nu_{\gamma, \epsilon}^{M}$ for cutoff potentials) [3], which reads in our model

$$
\nu_{\gamma}^{M} \equiv \int_{|\boldsymbol{\beta}|=1} b(|\boldsymbol{\alpha} \cdot \boldsymbol{\beta}|)|\boldsymbol{\alpha} \cdot \boldsymbol{\beta}|^{2} d \boldsymbol{\beta}=\int_{|\boldsymbol{\beta}|=1}|\boldsymbol{\alpha} \cdot \boldsymbol{\beta}|^{\gamma+2} d \boldsymbol{\beta}=\nu_{\gamma+2}, \nu_{\gamma, \epsilon}^{M}=\nu_{\gamma+2, \epsilon}
$$

When $\gamma \geq-2$, the difference $\left|\nu_{\gamma, \epsilon}^{M}-\nu_{\gamma}^{M}\right|$ is evaluated as

$$
\left|\nu_{\gamma, \epsilon}^{M}-\nu_{\gamma}^{M}\right|=4 \int_{0}^{\epsilon / 2}|\sin \varphi|^{\gamma+2} d \varphi \leq 4 \int_{0}^{\epsilon / 2} d \varphi=2 \epsilon \ll 1 .
$$

Therefore, the comparisons in Sec. 4.2 are reasonable. However, this estimate does not apply to $\gamma<-2$. In particular, when $\gamma$ is close to $-3, \nu_{\gamma, \epsilon}^{M}$ largely depends on $\epsilon$, so do the effective collision frequency and relaxation time; naive direct comparisons become improper. Figure 5.1 shows this situation for $\gamma=-2.5$ and -2.9 , illustrating the large impact of the cutoff parameter $\epsilon$ on the results. In these examples, the ratio of the momentum-transfer cross-section for the cutoff model with $\epsilon=\pi / 100$ to the noncutoff model is $\nu_{-2.5, \pi / 100}^{M} / \nu_{-2.5}^{M}=0.9044$ and $\nu_{-2.9, \pi / 100}^{M} / \nu_{-2.9}^{M}=0.3817$. Rescaling with a common effective relaxation time should be conducted in the range $\gamma<-2$. 


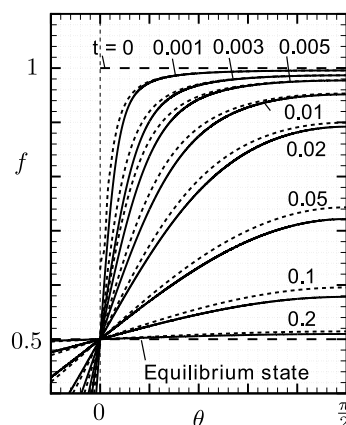

(a) $\gamma=-2.5$

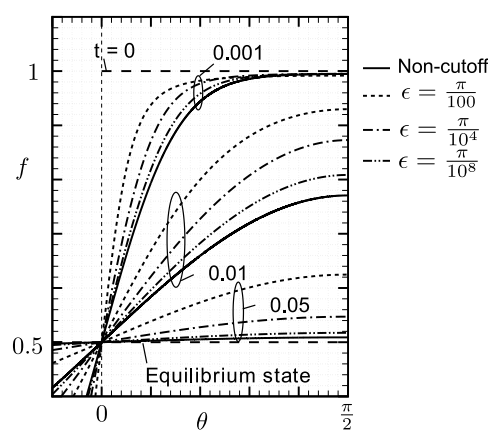

(b) $\gamma=-2.9$

Fig. 5.1 Comparisons between the non-cutoff and the cutoff model for $\gamma=-2.5,-2.9$ without rescaling of time. (a) $\gamma=-2.5$ and (b) $\gamma=-2.9$. In (a) dashed lines indicate the cutoff model with $\epsilon=\pi / 100$, while solid lines the non-cutoff model.

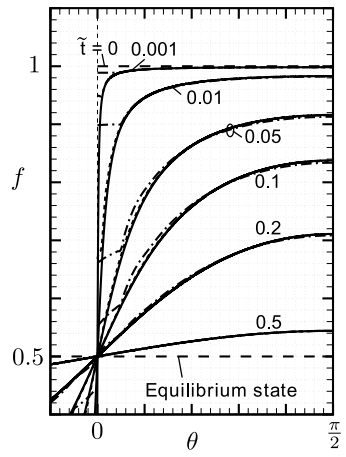

(a) $\gamma=-2$

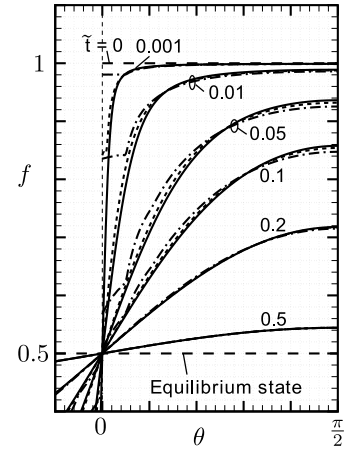

(b) $\gamma=-2.5$

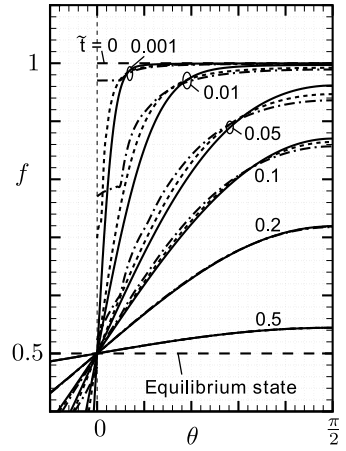

(c) $\gamma=-2.9$

Fig. 5.2 Comparisons between the non-cutoff and the cutoff model for $\gamma<-2$ after the rescaling of time: (a) $\gamma=-2$, (b) $\gamma=-2.5$, (c) $\gamma=-2.9$. Solid lines, dashed lines, and dashdotted lines indicate the results for $\epsilon=0$ (non-cutoff model), $\pi / 100$, and $\pi / 20$, respectively. The series in (4.2) is truncated at $m=1000$ for the non-cutoff model for $\gamma=-2$ and at $m=100$ otherwise.

In Fig. 5.2, we make comparisons of data at a common rescaled time $\tilde{t}$ defined as

$$
\nu_{1}^{M} \tilde{t}=\nu_{\gamma, \epsilon}^{M} t
$$

where $\nu_{1}^{M}$ is the momentum-transfer cross-section for the hard-disk potential without cutoff $(\gamma=1$ and $\epsilon=0)$. Comparisons in Fig. 5.2 for $\gamma=-2,-2.5,-2.9$ demonstrate that the above rescaling is indeed effective and, moreover, highlight the details of the cutoff effect for $\gamma<-2$ :

1. As in the case $\gamma>-2$, there appears a steeply varying part around $\theta=0$ for the non-cutoff model. However, the steepness is rather milder than before.

2. The cutoff still largely affects in the angular range $|\theta|<\epsilon$ and replaces the steep variation there with a jump discontinuity. However, due to the milder steepness, these effects are limited to the case of large cutoff parameter $(\epsilon=\pi / 20)$ or to the time duration very close to the initial time. 
Table 1 Main results for non-cutoff potentials

\begin{tabular}{|c|c|c|c|c|}
\hline & $\nu_{\gamma}$ & $\nu_{\gamma}^{M}$ & discontinuity of $f(t>0)$ & $\lim _{\theta \downarrow 0} \frac{\partial f}{\partial \theta}(t>0)$ \\
\hline \hline $0 \leq \gamma \leq 1$ & finite & finite & remains & finite \\
\hline$-1<\gamma<0$ & finite & finite & remains & $\infty$ \\
\hline$\gamma=-1$ & $\infty$ & finite & disappeared & $\begin{array}{c}\infty \text { for a finite time } \\
\text { and finite thereafter }\end{array}$ \\
\hline$-3<\gamma<-1$ & $\infty$ & finite & disappeared & finite \\
\hline
\end{tabular}

Table 2 Main results for the cutoff effect.

\begin{tabular}{|c|c|c|c|}
\hline & $\nu_{\gamma, \epsilon}^{M}-\nu_{\gamma}^{M}$ & discontinuity of $f(t>0)$ & influence \\
\hline \hline $0 \leq \gamma \leq 1$ & $O(\epsilon)$ & remains & very small \\
\hline$-2 \leq \gamma<0$ & $O(\epsilon)$ & remains & limited to $|\theta|<\epsilon$ \\
\hline$-3<\gamma<-2$ & sensitive to $\epsilon$ & remains & whole range of $\theta$ \\
\hline
\end{tabular}

3. In sharp contrast with $\gamma>-2$, the cutoff affects in the full range of angle $\theta$. However, the influence tends to vanish before reaching the final equilibrium state.

The last feature implies that the tentative conclusion in the last paragraph of Sec. 4.2 does not apply beyond the critical value of $\gamma=-2$. This is because the grazing collisions occupy an appreciable or possibly large portion in the total momentum exchange and accordingly neglecting or non-neglecting them should affect the whole relaxation process by collisions. The parameter range $-3<\gamma<-2$ is the transition regime toward another regime that the grazing collision is exclusively dominant and the process is of the Landau and the Fokker-Planck type equation.

\section{Conclusion}

In the present paper, we have studied the feature of grazing collisions and the influence of cutoff for the time-evolution of the two-dimensional Lorentz gas from the initial data with a jump discontinuity. The case that the momentum-transfer cross-section is finite $(-3<\gamma \leq 1)$ is studied.

The non-cutoff potentials are classified into two groups: one with a finite collision cross-section $(-1<\gamma \leq 1)$ and the other with an infinite collision cross-section $(-3<$ $\gamma \leq-1)$. For the former, the jump discontinuity propagates in time for $t>0$, while it vanishes immediately and the distribution function $f$ is continuous for $t>0$ for the latter. Except for the jump $f$ is mild in $\theta$ for $\gamma \geq 0$, while $f$ varies steeply for $\gamma<0$ near $\theta=0$, the angle at which the initial data has a jump discontinuity. This difference comes from whether or not the integrand of the collision cross-section is singular. The degree of steepness changes in quality according to whether or not the collision crosssection is finite. The $\theta$-derivative diverges at $\theta=0$ when $\gamma>-1$, while it is finite and thus $f$ is smooth (at least $C^{1}$ ) for $\gamma<-1$. For the critical case $\gamma=-1, f$ has a jump accompanied with the $\theta$-derivative divergence in a finite duration from the initial time. As time goes on, the $\theta$-derivative divergence becomes weaker and weaker and $f$ becomes $C^{1}$ after a critical instance. These are summarized in Table 1.

As to the cutoff potential, when $\gamma \geq-2$, the influence of the cutoff is limited in the range $|\theta|<\epsilon$, where $\epsilon$ is the cutoff parameter. For $\gamma \geq 0$, since $f$ for the non-cutoff model does not have a steeply varying part, the cutoff has essentially no effect. In contrast, it influences a lot for $-2 \leq \gamma<0$ and replaces the steep variation 
with a jump discontinuity. The feature of the cutoff effect for $\gamma \geq-2$ supports the interpretation that the difference of the mathematical property of $f$ between the cutoff and the non-cutoff potential is just the difference of modeling of the same phenomenon. However, for $-3<\gamma<-2$ the cutoff affects the results over the full range of angle $\theta$, which means the essential difference of the cutoff and the non-cutoff model in this regime. This is due to that the grazing collisions have an appreciable or large portion in the total momentum exchange, so that their omission is no longer appropriate in that regime. These features are summarized in Table 2.

\section{References}

1. C. Cercignani, The Boltzmann Equations and Its Applications, Springer, Berlin, 1998.

2. Y. Sone, Molecular Gas Dynamics, Birkhäuser, Boston, 2007.

3. G. A. Bird, Molecular Gas Dynamics and the Direct Simulation of Gas Flows, Clarendon, Oxford, 1994.

4. Y. Sone and H. Sugimoto, Strong evaporation from a plane condensed phase, in Adiabatic Waves and in Liquid-Vapor Systems, G. E. A. Meier \& P. A. Thompson eds., Springer, Berlin, 1990, pp. 293-304.

5. H. Sugimoto and Y. Sone, Numerical analysis of steady flows of a gas evaporating from its cylindrical condensed phase on the basis of kinetic theory, Phys. Fluids A 4, 419-440, 1992 .

6. Y. Sone and S. Takata, Discontinuity of the velocity distribution function in a rarefied gas around a convex body and the $\mathrm{S}$ layer at the bottom of the Knudsen layer, Transp. Theory Stat. Phys. 21, 501-530, 1992.

7. S. Takata, Y. Sone, and K. Aoki, Numerical analysis of a uniform flow of a rarefied gas past a sphere on the basis of the Boltzmann equation for hard-sphere molecules, Phys. Fluids A 5, 716-737, 1993.

8. L. Desvillettes, About the regularizing properties of the non-cut-off Kac equation, Commun. Math. Phys. 168, $417-440,1995$.

9. C. Villani, A review of mathematical topics in collisional kinetic theory, in Handbook of Mathematical Fluid Dynamics, Vol. 1, S. Friedlander \& D. Serre eds., Elsevier, Amsterdam, 2002.

10. L. Desvillettes, C. Graham, and S. Méléard, Probabilistic interpretation and numerical approximation of a Kac equation without cutoff, Stochastic Process. Appl. 84, 15-135, 1999.

11. B. Lucquin-Desreux and S. Mancini, A finite element approximation of grazing collisions, Transp. Theory Stat. Phys. 32, 293-319, 2003.

12. L. Pareschi, G. Toscani, and C. Villani, Spectral methods for the non cut-off Boltzmann equation and numerical grazing collision limit, Numer. Math. 93, 523-548 (2003).

13. R. Alexandre, Y. Morimoto, S. Ukai, C.-J. Xu, and T. Yang, Regularizing effect and local existence for the non-cutoff Boltzmann equation, Arch. Ration. Mech. Anal. 198, 39-123, 2010 .

14. M. Le Bellac, F. Mortessagne, and G. G. Batrouni, Equilibrium and Non-Equilibrium Statistical Thermodynamics, Cambridge University Press, Cambridge, 2004.

15. S. Takata, Invitation to the kinetic theory, Nagare, 27, 387 - 396 (2008). (in Japanese)

16. M. Abramowitz and I. A. Stegun, Handbook of Mathematical Functions, Dover, New York, 1972 .

17. S. Moriguchi, K. Udagawa, and S. Hitotumatsu, Iwanami Sugaku Kousiki III, Iwanami, Tokyo, 1960. (in Japanese)

18. G. B. Folland, Fourier Analysis and Its Applications, AMS, Providence, 1992. 